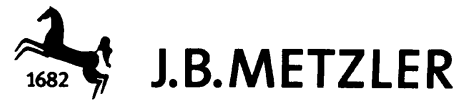


Benedikt Jeßing und Ralph Köhnen

\section{Einführung in die Neuere deutsche Literaturwissenschaft}

2., aktualisierte und erweiterte Auflage

Mit 39 Abbildungen

Verlag J. B. Metzler Stuttgart · Weimar 


\section{Die Autoren}

Benedikt Jeßing, geb. 1961; Studium der Fächer Deutsch und Biologie in Essen; 1991 Promotion; 2003 Habilitation; Studienrat im Hochschuldienst an der RuhrUniversität Bochum; bei J.B. Metzler ist erschienen: »Johann Wolfgang Goethe«, Sammlung Metzler 288, 1995; »Metzler Goethe-Lexikon", 22004 (Mitherausgeber); "Metzler Autorenlexikon", ${ }^{3} 2004$ (Mitherausgeber).

Benedikt Jeßing verfasste die Kapitel: Vorwort, 2.1, 2.2, 3.1, 3.2, 3.4, 3.5, 4, 6.1, 6.7, 6.11., 7. und 8 .

Ralph Köhnen, geb. 1961; Studium der Germanistik, Anglistik und Kunstgeschichte in Bochum, Berlin und München; 1994 Promotion; 2006 Habilitation; Studienrat im Hochschuldienst an der Ruhr-Universität Bochum. Veröffentlichungen zu Literaturgeschichte und -theorie vom 18. bis 20. Jahrhundert, zur Fachdidaktik sowie zu Komparatistik und Gegenwartskunst.

Ralph Köhnen verfasste die Kapitel: 1., 2.3, 2.4, 3.3, 5, 6.2 bis 6.6 und 6.8 bis 6.10

Bibliografische Information Der Deutschen Nationalbibliothek

Die Deutsche Nationalbibliothek verzeichnet diese Publikation in der Deutschen Nationalbibliografie; detaillierte bibliografische Daten sind im Internet über $<$ http://dnb.d-nb.de> abrufbar.

ISBN 978-3-476-02142-7

ISBN 978-3-476-05046-5 (eBook)

DOI 10.1007/978-3-476-05046-5

Dieses Werk einschließlich aller seiner Teile ist urheberrechtlich geschützt. Jede Verwertung außerhalb der engen Grenzen des Urheberrechtsgesetzes ist ohne Zustimmung des Verlages unzulässig und strafbar. Das gilt insbesondere für Vervielfältigungen, Übersetzungen, Mikroverfilmungen und die Einspeicherung und Verarbeitung in elektronischen Systemen.

(C) 2007 Springer-Verlag GmbH Deutschland Ursprünglich erschienen bei J.B. Metzler'sche Verlagsbuchhandlung und Carl Ernst Poeschel Verlag GmbH in Stuttgart 2007 www.metzlerverlag.de info@metzlerverlag.de 


\section{Inhaltsverzeichnis}

1. Einleitende Fragestellungen und Grundbegriffe ................ 1

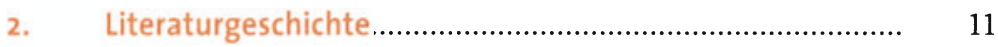

2.1 Terminologisches: Epochenbegriffe .................................. 11

2.2 Von der Reformation bis zur Französischen Revolution....... 13

2.2.1 Literatur der Renaissance und des Humanismus:

Zwischen Reformation und dem Beginn des Dreißigjährigen Krieges........................................................... 13

2.2.2 Die Literatur des 17. Jahrhunderts: Barock......................... 21

2.2.3 Literatur des 18. Jahrhunderts: Aufklärung ....................... 31

2.3 Von der Französischen Revolution bis zum Ersten Weltkrieg ....................................................... 47

2.3.1 Reaktionen auf die Französische Revolution: Romantik..... 47

2.3.2 Krise der Romantik und das Biedermeier ........................... 54

2.3.3 Frühe Formen des Realismus und Vormärz ....................... 58

2.3.4 Nach 1848: Der poetische Realismus ................................ 64

2.3.5 Naturalismus und Jahrhundertwende............................... 73

2.3.6 Expressionismus........................................................ $\quad 82$

2.4 Vom Ersten Weltkrieg bis zur Gegenwart.......................... 91

2.4.1 Die Avantgarden nach dem Ersten Weltkrieg..................... 91

2.4.2 Literarische Extreme: Weimarer Republik, Österreich,

2.4.3 Das 'Dritte Reich und die unmittelbare Nachkriegszeit ...... 100

2.4.4 Literatur der DDR.......................................................... 104

2.4.5 Literatur im Westen: Bundesrepublik, Österreich, Schweiz 113

2.4.6 \Postmoderne (.............................................................. 123

3. Literarische Gattungen .................................................. 135

3.1 Terminologisches: Gattungsbegriffe............................... 135

$3.2 \quad$ Lyrik ................................................................. 137

3.2.1 Zum Begriff der Lyrik ..................................................... 137

3.2.2 Formelemente und Formen der Lyrik ................................ 138

3.2.3 Zur Geschichte der Gattung: Verschiedene Konzeptionen von Lyrik.................................................................... 149

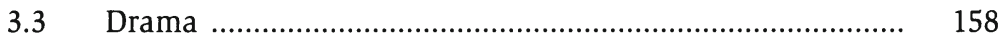

3.3.1 Probleme des Dramas .................................................. 158

3.3.2 Bauelemente des Dramas und dramaturgische Begriffe ...... 159

3.3.3 Theorie: Wirkungsabsichten des Dramas........................... 165

3.3.4 Historische Untergattungen............................................. 171

3.4 Erzählende Prosa ............................................................. 183

3.4.1 Epik - Erzählen - Erzählende Prosa .................................. 183

3.4.2 Strukturelemente des Erzählens .................................... 185 
3.4.3 Gattungen erzählender Prosa ........................................... 195

3.5 Literarische Gebrauchsformen ...................................... 206

4. Rhetorik, Stilistik und Poetik ........................................... 215

4.1 Terminologisches: Stilistische und poetologische Fachbegriffe.

4.2 Rhetorik und Poetik...................................................... 217

4.3 Rhetorik und literarische Stilistik .................................... 225

5. Literatur und andere Künste: Formen der Intermedialität .. 235

5.1 Methodologie und Begriffe .................................................. 235

5.2 Literatur und bildende Kunst............................................. 240

$5.3 \quad$ Literatur und Musik...................................................... 247

5.4 Gesamtkunstwerk ........................................................ 254

5.5 Literatur und Film ....................................................... 260

$5.6 \quad$ Literatur und Radio ......................................................... 266

6. Literaturwissenschaftliche Methoden und Theorien .......... 271

6.1 Zur Fachgeschichte der Neueren deutschen Literaturwissenschaft .................................................................. 271

$6.2 \quad$ Hermeneutik .............................................................. 278

6.2.1 Verstehen als Problem ....................................................... 278

6.2.2 Geschichte und Positionen der Hermeneutik .................... 278

6.3 Formanalytische Schule ................................................. 291

6.4 Rezeptionsästhetik ........................................................ 296

6.5 Psychoanalytische Literaturwissenschaft .......................... 302

6.5.1 Freuds Grundlagenbegriffe ......................................... 302

6.5.2 Anwendungen in Literatur- und Nachbarwissenschaften ... 303

6.6 Strukturalismus, Poststrukturalismus, Dekonstruktion...... 307

6.6.1 Strukturalismus.......................................................... 307

6.6.2 Poststrukturalismus ...................................................... 311

6.6.3 Dekonstruktion ............................................................. 314

6.7 Sozialgeschichte der Literatur/Literatursoziologie .............. 319

6.7.1 Vorgeschichte................................................................ 319

6.7.2 Analysegegenstände und zentrale Fragestellungen ............ 322

6.7.3 Ende und/oder Nachgeschichte sozialgeschichtlicher Literaturwissenschaft ................................................. $\quad 326$

6.8 Diskursanalyse ........................................................... 328

6.8.1 Begriffsentwicklung ...................................................... 328

6.8.2 Michel Foucault: Grundlegungen des Diskursbegriffs ....... 328

6.8.3 Foucaults Institutionenkritik und Machtanalytik ............. 330

6.8.4 Anwendungsmöglichkeiten in der Literaturwissenschaft.... 332

6.8.5 Perspektiven und Kritik................................................. 335

6.9 Systemtheorie ............................................................. 338

6.9.1 Begriffsentwicklung .................................................... 338

6.9.2 Niklas Luhmann: Grundbegriffe ..................................... 339 
6.9.3 Vorgänge des Beobachtens .............................................. 341

6.9.4 Wechselwirkung zwischen Literatur und anderen Systemen...................................................................... 342

6.9.5 Literarische Selbstreferenzialität als Systembildung .......... 343

6.9.6 Perspektiven für die Literaturwissenschaft und Kritik ....... $\quad 345$

6.10 Medienwissenschaften ................................................ 348

6.11 Kulturwissenschaftliche Ansätze................................... 357

6.11.1 Cultural Studies .............................................................. 357

6.11.2 Feministische Literaturtheorie/Gender Studies ................. 360

6.11.3 New Historicism ............................................................. 364

6.11.4 Anthropologie ........................................................ 366

7. Literaturwissenschaftliche Praxis ...................................... 371

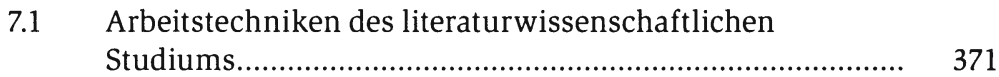

7.2 Editionsphilologie........................................................ 379

7.3 Berufsfelder für Germanist/innen ................................ 386

8. Anhang ......................................................................... 395

$8.1 \quad$ Abkürzungen.............................................................. 395

$8.2 \quad$ Bibliographie .......................................................... 395

8.3 Personen register ............................................................ 403

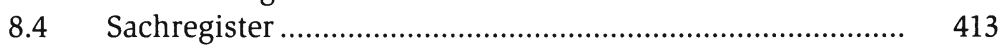




\section{Vorwort}

In einem Brief an den Freund, Musiker und Schriftsteller Johann Friedrich

Rochlitz teilt Goethe das Lesepublikum seiner Zeit in drei Gruppen ein:

Es giebt dreierley Arten Leser: Eine, die ohne Urtheil genießt, eine dritte, die ohne zu genießen urtheilt, die mittlere, die genießend urtheilt und urtheilend genießt; diese reproducirt eigentlich ein Kunstwerk auf's neue. Die Mitglieder dieser Classe [...] sind nicht zahlreich, deshalb sie uns auch werther und würdiger erscheinen.

Die Kriterien für diese Dreiteilung des Lesepublikums sind, in unterschiedlicher Zusammensetzung, "Urtheil«, also kritisches Unterscheidungsvermögen, und "Genuß«, also sinnliche Erfahrung. Lesen setze, so Goethe, beides voraus: Bloßer Genuss, sinnliches ,Verschlingen der Bücher, sei ebenso wenig Lesen wie der rein kritisch-analytische Blick. Und gelungenes Lesen sei viel mehr als Genuss und Urteil, nämlich die nochmalige Herstellung des literarischen Kunstwerks selbst, sei es als einfühlendes Nacherleben, kongenialer Nachvollzug oder konstruktive Eigenschöpfung.

Gleichgültig, ob heute diese Vorstellung vom idealen Leser noch geteilt werden kann oder nicht - die Befähigung zu einem Umgang mit Literatur, der ihrer Komplexität ebenso angemessen ist wie ihrem Kunstcharakter, ist in unseren Tagen ebenso wünschenswert wie zur Zeit Goethes. Literatur ist, emphatisch gesprochen, eine spezifische Form kulturellen Gedächtnisses; die intensive Beschäftigung mit Literatur ist gesellschaftliche Erinnerungsarbeit. Und Literatur ist allen anderen Formen des kollektiven Erinnerns überlegen: Denn nirgends sonst werden geschichtliche Erfahrungen in so hohem Maße gleichzeitig vergesellschaftet und radikal individualisiert versprachlicht sowie in ästhetische Form gebracht wie in der Literatur. In literarischen Texten sprechen Individuen, Autorinnen und Autoren; wie sie sprechen, ist aber nicht nur individuell bedingt, sondern über die Sprache und die Vielzahl stilistischer und literarischer Traditionen, die sie benutzen, mit der Gesellschaft und der Geschichte verbunden.

Hier hat die Neuere deutsche Literaturwissenschaft ihren Sitz im Leben: Sie ist die Universitätsdisziplin, durch die die Techniken und Mittel weitergegeben werden, sich diese Wissensbestände anzueignen und so das kulturelle Gedächtnis zu verlängern. Texte aus dem 16., dem 18. oder dem 20. Jahrhundert zu lesen, sie zu verstehen oder sie verstehen zu lernen, sie zu ‘deuten im Kontext ihres eigenen Horizonts oder auch im Horizont heutiger Leser/innen - all dies erst setzt die kulturelle Überlieferung fort.

Brief Goethes an Joh. Fr. Rochlitz vom 13.6 .1819 
Die wissenschaftliche Beschäftigung mit Literatur setzt beide von Goethe thematisierten Wahrnehmungskategorien voraus: sinnliches Vermögen, Genuss und Anteilnahme ebenso wie ein wissenschaftliches Analyseinstrumentarium, kritisches Unterscheidungs- und Urteilsvermögen. Anteilnahme an literarischen Texten lässt sich nicht lernen, vor dem Beginn des Studiums der Literaturwissenschaft sollte jede(r) Studierende sich selbst vergewissern, ob sie bzw. er überhaupt gerne (viel und intensiv) liest! Das Studium geht natürlich weit über das Lesen hinaus: Es vermittelt die wissenschaftlichen Beschreibungs- und Analyseverfahren, die zu einer plausiblen Deutung eines literarischen Textes führen, zu einem begründeten Urteil.

Unser Band stellt dieses literaturwissenschaftliche Handwerkszeug systematisch vor, das heißt, er bildet in seiner Gliederung das Profil der Neueren deutschen Literaturwissenschaft nach ihren Gegenständen, Verfahren, Methoden und ihrer Terminologie ab und beabsichtigt, ohne im Spektrum der Methoden und Zugangsweisen selbst Position zu beziehen, die Bandbreite literaturwissenschaftlicher Reflexionsgegenstände annähernd neutral zu präsentieren:

Gegenstände der Literaturwissenschaft

Das Ziel dieser Einführung ist ein umfassender Überblick über das Grundwissen der Neueren deutschen Literaturwissenschaft, der die Einführungsveranstaltung im ersten Studiensemester begleiten und die eventuelle Abschlussklausur vorbereiten helfen soll. Darüber hinaus aber sollen bis zur Examensvorbereitung relevante grundlegende Orientierungen in den verschiedenen Abteilungen des Faches bereitgestellt werden. Das Ziel wäre jedenfalls erreicht, wenn die Leserin oder der Leser dieses Buches sich besser in den Stand gesetzt fühlte, über Literatur 'genießend urteilen zu können und sie gleichzeitig urteilend zu genießen s sowie Literaturerfahrung in verschiedenste Formen mündlicher und schriftlicher Mitteilung übersetzen zu können: in ein Statement oder ein Kurzreferat, in Moderation, Podiumsdiskussion oder Streitgespräch, schriftliche Hausarbeit, Lexikonartikel oder Literaturkritik - also, um noch einmal mit Goethe zu sprechen, "das literarische Kunstwerk auf's neue zu reproduciren". 
Jedes Kapitel dieser Einführung wird mit einem Literaturverzeichnis abgeschlossen, in der, neben der im Text zitierten, die grundlegende und weiterführende Fachliteratur zum Thema aufgeführt ist. Auf die in dieser Bibliographie genannten Titel wird im Text verwiesen in der Kurzform (Autorname, Jahreszahl, Seite).

Darüber hinaus versammelt die Abschlussbibliographie weitere Fachliteratur, die als Handwerkszeug des literaturwissenschaftlichen Studiums unverzichtbar ist: Wichtige Literaturlexika, die prominenten mehr- und einbändigen Literaturgeschichten, weitere Einführungen in die Neuere deutsche Literaturwissenschaft, in die Textanalyse und die Bücherkunde, die zentralen periodisch erscheinenden und abgeschlossenen Fachbibliographien, die Titel der wichtigsten Fachzeitschriften sowie hilfreiche Internetseiten. Schließlich ermöglichen ein Personen- sowie ein Sachregister die gezielte Arbeit mit dem Band.

Die 2. Auflage unseres Einführungsbandes möchte mit ihrem neuen, stärker strukturierten, aufgelockerten und durchsichtigeren Erscheinungsbild die Gegenstände der Neueren deutschen Literaturwissenschaft weiterhin breit darstellen, aber auch deutlicher innerhalb dieses Wissensraums orientieren. In chronologischen Werklisten soll eine Auswahl der jeweils wichtigsten bzw. einschlägigen literarischen Werke einer Epoche der Gattung übersichtlich dargestellt werden.

Die Fragen bzw. Arbeitsaufgaben an den Kapitelenden sind teilweise relevant für Abschlussklausuren in vielen Grundkursmodellen, teilweise als weiterführende Fragen gestellt.

Antworten bzw. Ausführungen dazu sind auf den homepages der Autoren zu finden, und zwar unter http://homepage.ruhr-uni-bochum.de/ benedikt.jessing/Einfuehrung-NDL.html und http://homepage.ruhr-unibochum.de/ralph.koehnen/Einfuehrung-NDL.html. 